\title{
A Numerical Study on the Resistance Prediction of a Medium Speed Craft
}

\author{
Shao-Quan DENG and Guo-Xiang HOU ${ }^{a}$ \\ School of Naval Architecture and Ocean Engineering, Huazhong University of Science and \\ Technology, Wuhan, China \\ ahouguoxiang@163.com
}

Keywords: Ship resistance prediction, CFD, Navigation attitude, Hydrostatic equilibrium equations of ship.

\begin{abstract}
This paper aims to study the resistance performance of a medium speed ship, the steadystate Reynolds-averaged Navier-Stokes (RANS) simulations are performed by utilizing a commercial CFD software package named Fluent. With the increasing of the ship's speed, the navigation attitude changes significantly as well, in order to achieve precise prediction results, the sinkage and trim motion should be taken into consideration. The author adopted a simple but effective method called "static equilibrium method" to determine the sinkage and trim motion and adjust the navigation attitude. Compared to the result from the towing tank experiment, the predicted result shows a good agreement, it indicates that this method can be a reliable method to predict the resistance performance of this kind of ships and can be used in the process of designing ships.
\end{abstract}

\section{Introduction}

The traditional way of researching the resistance performance of ships has always been the towing tank experiment and it's still widely used and indispensable now. However, in recent years, the computer hardware performance has enjoyed a tremendous progress as well as the numerical algorithms of the CFD method, as a consequence, the CFD method has become a commonly used and important tool to so many researchers in ship engineering area. Compared to the traditional experimental method, the CFD method is time-saving, low-cost, environmental-friendly; furthermore, we are able to obtain much more useful details about the flow distribution which may be difficult to measure in the experiment, and it may help the researchers gain a better understanding of the problems they study on.

The wave making problem was studied by using the free surface potential theory before. With the rapid development of the CFD method and computer hardware, the numerical simulation based on the RANS model has become a hot issue. After years of studying by all the researchers, numerous of achievement in RANS-based ship resistance simulation field has been reached. Alexandre T. P. Alho et al. [1] and Omar Yaakob et al. [2] tried to get the resistance by adopting a fixed ship model using the VOF method to calculate the free surface effect. H.C. Raven et al. [3] and Yi Wen-Bin et al. [4] provided CFD based-ways to predict the ship resistance in full scale. A different way of predicting resistance in full scale by Ni Chong-Ben et al. [5], in their method, ship form factor is obtained by solving the viscosity resistance of double-model in turbulence flow theory and the wave making resistance in ideal flow theory. Tahsin Tezdogan et al. [6] and Guo Chun-Yu et al. [7] employed the dynamic mesh model and 6 DOF model in order to evaluate the resistance take the sinkage and trim effect into consideration. Dong Wen-Cai et al. [8] proposed a method to predict the sinkage and trim motion without adopting the dynamic mesh model and 6 DOF model. Factors effecting ship resistance calculation are also studied by Deng Rui et al. [9] and Sun Hua-Wei et al. [10].

In this paper, the resistance of a medium speed ship was being studied. The fixed ship model was employed when the ship is travelling forward at relatively low Froude Numbers $(\mathrm{Fn}<0.3)$; but with the increasing of Fn, the sinkage and trim motion effects play important roles in resistance prediction, consequently, the fixed model is not applicable anymore, under these circumstances, a new method 
which can take the sinkage and trim effect into consideration needs to been introduced. Here the author applied a "static equilibrium method" to solve this problem, which turned out to be a simple but rather effective method.

\section{Geometry and Grid Descriptions}

\section{Details of the Geometry}

The calculated ship model in question is the same size of the physical model which was being studied in the towing tank experiment so that we can make a convenient result comparison between them.

The applied model is a bare ship hull without any appendages or propellers. The model-scale hull properties are presented in Table 1. Fig. 1 illustrates the hull sections of the ship in question. And we can have a view of the 3-dimensional model from Fig.2.

Table 1. Model-scale ship properties.

\begin{tabular}{|c|c|}
\hline Property & Model \\
\hline Length between the perpendiculars $\left(L_{\mathrm{PP}}\right)(\mathrm{m})$ & 3.500 \\
\hline Beam $(B)(\mathrm{m})$ & 0.625 \\
\hline Design draft $(T)(\mathrm{m})$ & 0.235 \\
\hline Displacement $(\Delta)\left(\mathrm{m}^{3}\right)$ & 0.2441 \\
\hline Longitudinal centre of buoyancy $(\mathrm{LCB})(\mathrm{m})$ & 1.549 \\
\hline
\end{tabular}

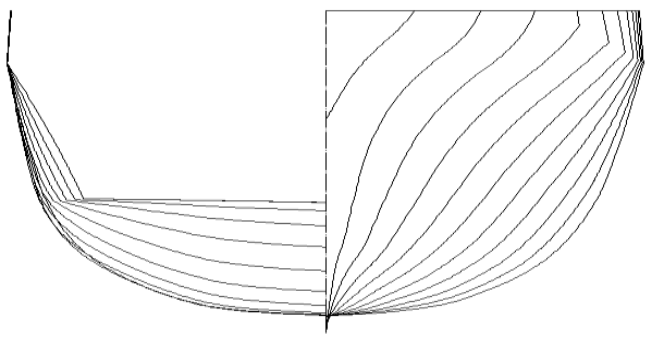

Fig. 1.Hull sections of model ship

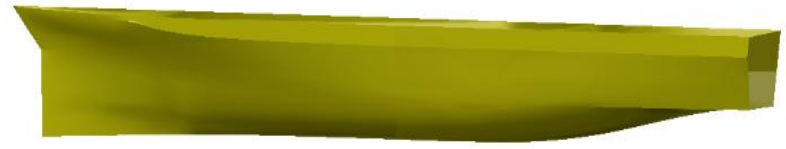

Fig. 2. A three-dimensional view of the model ship

\section{Discussion of the Grid Configuration}

As discussed in so many literatures, the grid generation plays an absolutely important role in a successful and precisely simulation. For a given mathematical model and a specified numerical approach, numerical errors are mainly determined by grid generation [1]. Obviously, adequate attention needs to be paid to this part.

The computation domain was set to be a rectangular shape zone. Half of the longitudinal symmetrical ship was chosen as the analysis object by employing the symmetry boundary at the plane of symmetry so as to reduce the computation cost. The length, width, depth of the domain is $6 L, 1 L$, and $1.5 L$ respectively, where $L$ is the length of ship. The length of the upstream region is $1 L$ and the downstream region is $4 L$. The water depth is $1 L$ and the height of the air region is $0.5 L$.

In this essay, grid generation was performed in the ICEM CFD software. The whole solution domain was discretised with fine-quality multi-block and body-fitted structured grids, usually of $\mathrm{H}$ $\mathrm{O}$ topology. Structured grids based on hexahedral elements enjoy a considerable advantage over other grid methods. Compared to the tetrahedral grid, the structured grid method costs much less elements 
to cover a same region with the same element size. What's more, it enjoys a better convergence performance.

In order to simulate this case more accurate, the computation grid had areas of well-refined grid size in the area near the ship hull surface as well as the free surface zone. The prism boundary layer grid is adopted around the ship surface. In this paper, the $\mathrm{y}^{+}$value around the wetted surface of the ship model is basically within the range of 11.5-200, which is claimed to be an appropriate range for resistance simulation project [9]. Besides, for the reason of decreasing the number of total elements and the computing time, gradual-changed grid size was adopted, the size of grid slowly increase as it getting far away from the ship hull and free surface region. With key points mentioned before, the flow field can be well simulated and the free surface can be captured well. About 1.5M hexahedral elements were generated in the domain. Fig.3 and Fig.4 depicted the mesh distribution of the computation model.

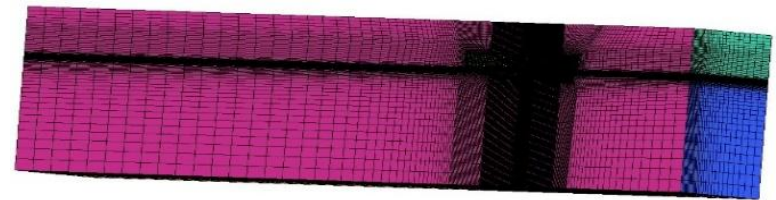

Fig. 3. General view of the volume mesh of the computational domain

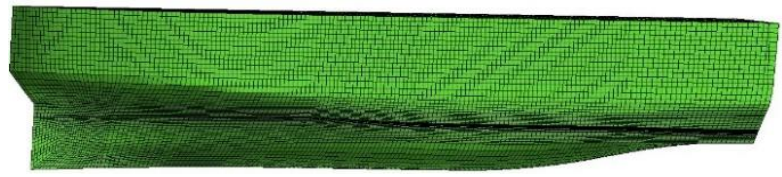

Fig. 4. Mesh distribution of the hull surface

\section{Numerical Modelling}

In this section, the details of the numerical simulation approaches used in this study will be provided and discussed.

\section{Governing Equations}

In the present work, the equations governing the problem of the flow around the ship hull were solved using the commercial code Fluent. The RANS equations are solved by a finite volume approach. The continuity and momentum equations used for incompressible turbulent flow can be expressed as Eq. 1 .

$$
\left\{\begin{aligned}
\frac{\partial u_{i}}{\partial x_{i}}=0 & \\
\rho \frac{\partial u_{i}}{\partial t}+\rho \frac{\partial u_{i} u_{j}}{\partial x_{j}}= & -\frac{\partial p}{\partial x_{i}}+\frac{\partial}{\partial x_{j}}\left[\mu\left(\frac{\partial u_{i}}{\partial x_{j}}+\frac{\partial u_{j}}{\partial x_{i}}\right)\right. \\
& \left.-\frac{2}{3} \mu \frac{\partial u_{l}}{\partial x_{l}} \delta_{i j}\right]+\frac{\partial}{\partial x_{j}}\left(-\rho \overline{u_{i}^{\prime} u_{j}^{\prime}}\right)+\rho f_{i}
\end{aligned}\right.
$$

Where $\rho$ is the density of fluid, $u_{i}$ denotes velocity component in $x_{i}$ direction, while $p$ is the static pressure of fluid, and $\mu$ is fluid viscosity; $-\rho \overline{u_{i}^{\prime} u_{j}^{\prime}}$ and $\rho f_{i}$ denote Reynolds stress which will be calculated by Boussinesq hypothesis and body forces per unit volume respectively.

\section{Turbulence Model}

The SST $k-w$ eddy viscosity based model developed by Menter was adopted for this simulation [11]. This method takes advantage of both the standard $k-w$ model and $k-\varepsilon$ model by blending the robust and accurate formulation of the $k-w$ model in the near-wall region with the free-stream independence of the $k-\varepsilon$ model in the far field. It shows a good applicability in ship resistance prediction field. 


\section{Volume of Fluid (VOF) Method}

In order to predict the wave-making resistance, Volume of Fluid (VOF) method was adopted for the numerical treatment of the free surface. The volume fraction equation for an incompressible fluid can be expressed as Eq. 2.

$$
\frac{\partial F}{\partial t}+\frac{\partial}{\partial x_{i}}\left(u_{i} F\right)=0
$$

Where, $F$ is a volume fraction function, $u_{i}$ is the Cartesian velocity components and $t$ is the variable time.

\section{Boundary Conditions and Numerical Scheme}

The boundary conditions should be defined based on the physics of the problem to be solved. Selecting the most appropriate boundary can prevent us from the unnecessary problems. As we can see from Fig. 5, a velocity inlet boundary condition was set in the positive $x$-direction; conversely, pressure outlet boundary was set in the negative $x$-direction, the pressure varied with the water depth is defined by the User Defined Function (UDF) facilities provided by Fluent. The slipping wall condition was applied to the side, top and bottom of the domain. And as mentioned before, the symmetry plane of ship in longitudinal direction is modelled as the symmetry boundary.

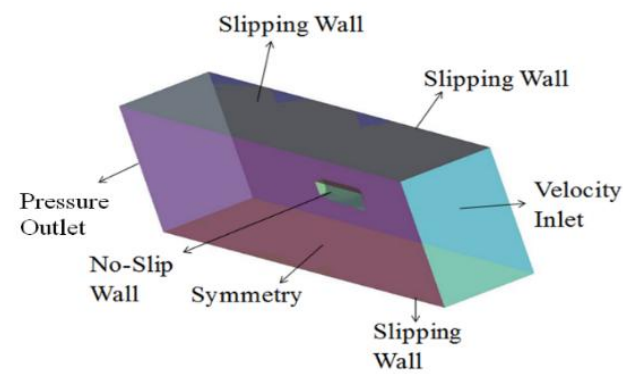

Fig. 5. General view of the boundary conditions

The steady state simulation is adopted; the overall solution procedure was obtained according to a SIMPLE-type algorithm. The volume fraction spatial discretization scheme was set to be implicit Modified HRIC to get the sharp interface between the two phases. And the rest of all convection terms and diffusion terms were discretized by applying second-order upwind scheme.

\section{Determination of Sinkage and Trim}

In this work, the navigating attitude is assessed in an estimation-modification way. In general, after once or twice modifications, we can obtain the ultimate navigation attitude, at which the hydrodynamic force and gravity force finally reach an approximate balance. The static equilibrium equations can be expressed as Eq. 3 .

$$
\left\{\begin{array}{l}
\sum F_{\mathrm{Z}}=0 \\
\sum M_{\mathrm{G}}=0
\end{array}\right.
$$

Where $F \mathrm{z}$ means the resultant acting on in $z$ direction; $M_{\mathrm{G}}$ is the resultant moment acting on the ship hull around the $y$ axis.

Considering that the trim and sinkage motion is relatively small. We can assess the trim and sinkage motion values by solving Eq. 4 .

$$
\left\{\begin{array}{l}
F_{\mathrm{ZH}}=G+\rho g S \Delta h \\
M_{\mathrm{GH}}=\Delta \overline{G M_{\mathrm{L}}} \sin \theta
\end{array}\right.
$$

Where $F_{\mathrm{ZH}}$ is the fluid-induced force in the $\mathrm{Z}$ direction; $G$ means the gravity force of the ship; $\rho$ is the density of water; $g$ is the gravity acceleration; $S$ is the area of the waterline section. $M_{\mathrm{GH}}$ is the 
fluid-induced resultant moment acting on the centre of gravity of the ship about the $y$-axis. $\Delta$ is the displacement of ship; $\overline{G M_{\mathrm{L}}}$ is the longitudinal metacentric height and $\theta$ is the trim angle.

$F_{\mathrm{ZH}}$ and $M_{\mathrm{GH}}$ can be obtained by integrating the fluid pressure acting on the ship hull. After the first simulation, we can achieve the first-time-estimated value of sinkage and trim. Then translate and rotate the ship hull surface and the corresponding blocks to the estimated navigation attitude position, and regenerate grids and recalculate in Fluent. And redo the same process mentioned before until the resultant of forces and moments acting on ship hull reach the approximate equilibrium (when the $\Delta h$, $\theta$ values both very close to 0 ).

This modification process won't be complicated and time-consuming since the mesh definition is stored in block files, it's really convenient to translate and rotate geometry and block files and then regenerate the new meshes in ICEM CFD software.

In fact, the dominated method to predict the navigation attitude at present is by employing the dynamic mesh methods to solve the dynamic fluid body interaction issue in the time domain, still the author want to say that the method adopted in this work can be a good choice to solve this kind of problems. Compared to the dynamic mesh method, we can adopt the steady-state simulation way to solve the problem, which is much time-saving to achieve the convergence, at least 3-5 times faster according to the running test of the author. Furthermore, the mesh quality can be well controlled in the steady state simulations and the meshes won't be changed in the simulation process so that we can get the result without any mesh quality trouble once you've got a fine mesh quality model. As we know, there is a big chance for the decreasing of mesh quality in the dynamic mesh model simulation, which will lead to bigger error propagation during the simulation; even worse, the simulation may be interrupted due to the generation of negative volume meshes. So you may have to pay a lot attention and spend much time trying to get the appropriate mesh distribution.

\section{Result Comparison and Discussion}

9 different velocities with the Fn in the range of 0.132 to 0.483 was studied in the numerical simulation as well as in the towing tank experiment. The physical experiment was carried out in the towing tank of Huazhong University of Science and Technology. The length, width and depth of the tank are $175 \mathrm{~m}, 6 \mathrm{~m}$ and $4 \mathrm{~m}$, respectively. The resistance, sinkage and Trim motion were measured during the experiment.

\section{Discussion of Resistance Prediction}

Table 2, Fig.6 and Fig.7 present the resistance comparisons among the experimental data $\left(R_{\mathrm{E}}\right)$, calculated data based on the fixed model which neglect the sinkage $\left(R_{\mathrm{F}}\right)$ and trim effects and the resistance calculation results of the attitude-modified model which take the sinkage and trim effects into consideration $\left(R_{\mathrm{M}}\right)$.

Table 2. The resistance results for all cases by CFD and EFD methods

\begin{tabular}{|c|c|c|c|c|c|c|}
\hline \multirow{2}{*}{$v_{\mathrm{m}}(\mathbf{m} / \mathbf{s})$} & \multirow{2}{*}{$\mathbf{F n}$} & \multicolumn{3}{|c|}{$\boldsymbol{R}_{\mathbf{t m}}(\mathbf{N})$} & \multicolumn{2}{c|}{ Error (\%) } \\
\cline { 3 - 7 } & & $\boldsymbol{R}_{\mathbf{E}}$ & $\boldsymbol{R}_{\mathbf{F}}$ & $\boldsymbol{R}_{\mathbf{M}}$ & $\boldsymbol{R}_{\mathbf{F}}$ & $\boldsymbol{R}_{\mathbf{M}}$ \\
\hline 0.772 & 0.132 & 4.336 & 4.504 & - & 3.87 & - \\
\hline 1.029 & 0.176 & 7.907 & 8.280 & - & 4.72 & - \\
\hline 1.286 & 0.219 & 13.557 & 13.612 & - & 0.40 & - \\
\hline 1.543 & 0.263 & 21.082 & 20.562 & - & -2.47 & - \\
\hline 1.800 & 0.307 & 30.431 & 28.624 & 30.708 & -5.94 & 0.91 \\
\hline 2.058 & 0.351 & 42.134 & 38.852 & 42.780 & -7.79 & 1.53 \\
\hline 2.315 & 0.395 & 63.853 & 57.018 & 64.630 & -10.70 & 1.22 \\
\hline 2.572 & 0.439 & 108.597 & 87.984 & 106.498 & -18.98 & -1.75 \\
\hline 2.829 & 0.483 & 159.108 & 120.516 & 161.246 & -24.26 & 1.34 \\
\hline
\end{tabular}


In the table above, $v_{\mathrm{m}}$ is the forward speed of model ship. Fn is the Froude number, which can be expressed as Fn $=\frac{v_{\mathrm{m}}}{\sqrt{g L}}$, the $R_{\mathrm{tm}}$ is the total resistance of the model. The relative error is calculated based on the experimental results, which can be calculated by $\frac{\left(R_{\mathrm{F}} \mathrm{or} R_{\mathrm{M}}-R_{\mathrm{E}}\right)}{R_{\mathrm{E}}} \times 100 \%$.

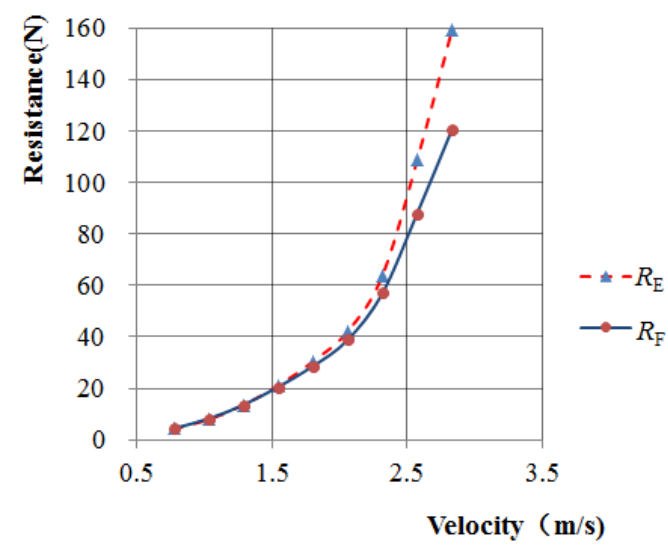

Fig. 6. Comparison of the resistance values obtained by Fixed Model simulation and EFD

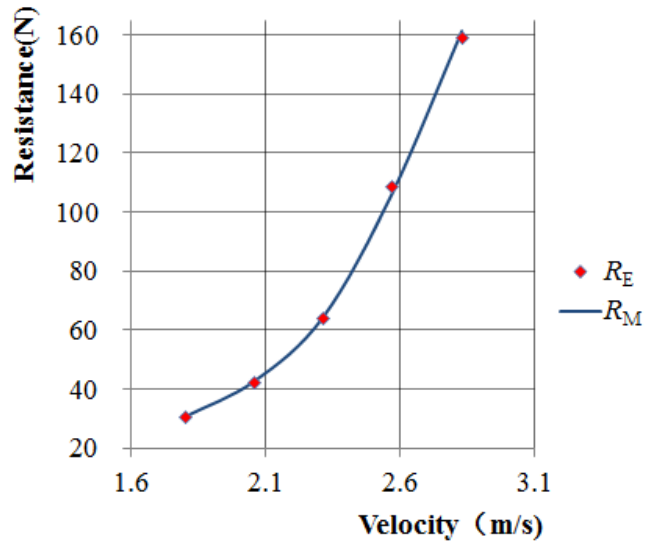

Fig. 7. Comparison of the resistance values obtained by Modified Model simulation and EFD

As we can see from Table 2 and Fig. 6, for the fixed model, the resistance results agree well with the experimental values when Fn $<0.3$ (that is when $v_{\mathrm{m}}$ smaller than $1.759 \mathrm{~m} / \mathrm{s}$ ), the calculation errors were less than 5\%, which are basically acceptable in practical engineering application, however, due to the obvious change of navigation gesture, the simulation error increases gradually with the increasing of $\mathrm{Fn}$, when $\mathrm{Fn}>0.3$, the error is always greater than $5 \%$, and even exceeds $20 \%$ when $\mathrm{Fn}=0.483$, which make the fixed model simulation results unreasonable and unacceptable. As a result, the sinkage and trim effects should be included in the simulation.

So the navigation attitude had been modified based on the "Static Equilibrium Method" introduced before when Fn exceeds 0.3. Good agreements had been reached when adopting the modified model to do the simulation, with the maximum resistance error no more than $2 \%$ at each of the corresponding Fn. It shows that the modification method proposed before can be an applicable approach in terms of ship resistance prediction.

\section{Discussion of the Sinkage and Trim Prediction}

Besides the resistance performance, the sinkage and trim motion were studied. As the velocity increases, the sinkage and trim motion, there is a big difference of gesture compare to the initial state (when Fn=0). We can see the details from Table 3, Fig.8 and Fig. 9. In Table 3, $\Delta$ means the difference between CFD-based value and EFD-based value. $S$ is the sinkage value, $\theta$ indicates the 
trim value, and is positive when trim by stern. The superscripts $\mathrm{E}$ and $\mathrm{M}$ represent the experimental results and the simulation results of the modified model, respectively.

Table 3. The sinkage values for all cases by CFD and EFD methods

\begin{tabular}{|c|c|c|c|c|c|c|c|}
\hline \multirow{2}{*}{$\begin{array}{c}\boldsymbol{v}_{\mathbf{m}} \\
(\mathbf{m} / \mathbf{s})\end{array}$} & \multirow{2}{*}{$\mathbf{F n}$} & \multicolumn{3}{|c|}{ Sinkage $(\mathbf{m m})$} & \multicolumn{3}{c|}{ Trim $\left(^{\circ}\right)$} \\
\cline { 3 - 8 } & & $\boldsymbol{S}_{\mathbf{E}}$ & $\boldsymbol{S}_{\mathbf{M}}$ & $\Delta \boldsymbol{S}_{\text {M-E }}$ & $\boldsymbol{\theta}_{\mathbf{E}}$ & $\boldsymbol{\theta}_{\mathbf{M}}$ & $\Delta \boldsymbol{\theta}_{\text {M-E }}$ \\
\hline 1.800 & 0.307 & -6.55 & -7.45 & -0.90 & -0.03 & 0.064 & 0.094 \\
\hline 2.058 & 0.351 & -10.44 & -10.80 & -0.36 & -0.07 & 0.085 & 0.155 \\
\hline 2.315 & 0.395 & -17.25 & -16.40 & 0.85 & 0.234 & 0.416 & 0.182 \\
\hline 2.572 & 0.439 & -22.42 & -24.41 & -1.98 & 1.016 & 1.104 & 0.088 \\
\hline 2.829 & 0.483 & -22.22 & -26.66 & -4.44 & 1.858 & 1.984 & 0.126 \\
\hline
\end{tabular}

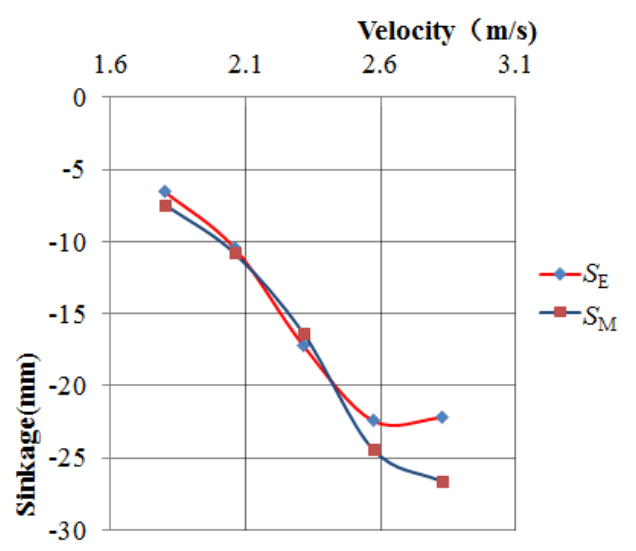

Fig. 8. Comparison of sinkage values obtained by CFD and EFD methods

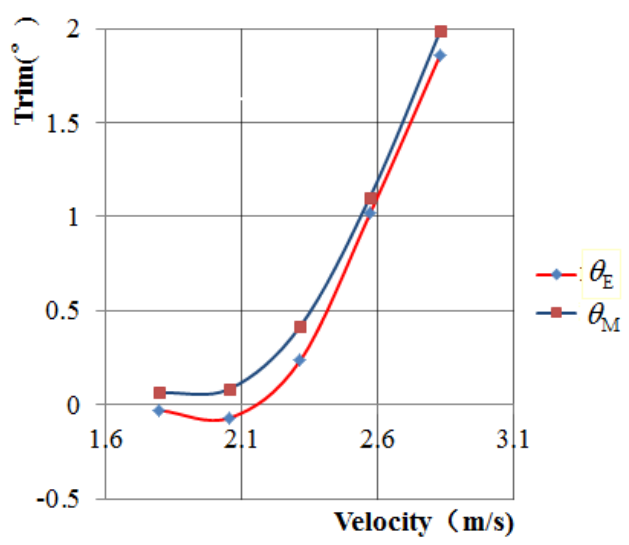

Fig. 9. Comparison of sinkage values obtained by CFD and EFD methods

As Table 3, Fig. 8 and Fig. 9 jointly show, the sinkage and trim increase with increasing Fn, or ship velocity; when the Fn is within the confines of 0.307 to 0.483 except when Fn $=0.483$, where the experimental sinkage value slightly decreases compare to the sinkage value when $\mathrm{Fn}=0.439$.

Both of the sinkage and trim prediction results from the numerical simulation roughly agree well with the experimental measurements, they have the similar variation tendency against various Fn compare to the EFD results. As to the sinkage performance, the maximum difference between the EFD and CFD results is $4.4 \mathrm{~mm}$, when $\mathrm{Fn}=2.829$. On the other hand, the maximum difference for the trim prediction is 0.182 , when $F n=0.395$. It should been noted that the sinkage and trim prediction are not as precise as the resistance prediction. The reason maybe as follows:

1) The difference may be caused due to the systematic error. For example, the simulation condition is an approximation to the experimental conditions; the RANS simulation is a simplified model of the real flow etc. 
2) There may be some tiny differences between numerical model and physical ship model.

3) The static-equilibrium-based method applied in this work can hardly predict the completely equilibrium state. In fact, the predicted equilibrium state here is not the exactly equilibrium state for the ship. There is still small unbalanced force acting on the modified model.

Further research may be needed to have a more clear understanding of this problem.

Despite the relatively greater errors in sinkage and trim prediction, the resistance prediction result has very good precision, which is the key goal of this simulation. So still this simulation method is adoptable in terms of resistance prediction.

\section{Conclusions}

This paper focused on the prediction of the flow around a medium speed ship and forecast the ship resistance at a range of Fn. The simulations were performed by a commercial CFD solver, Fluent. The sinkage and trim effects had been taken into consideration by utilising the method proposed in the paper. A comparison was made between the CFD and EFD results. The overall simulation results obtained suggest that the numerical model presented here can be applied to predict the physics of flow around the model ship hull.

The results show that the fixed model can be used when the ship forward speed is relatively small. In this work, if $F n<0.3$, the ship model can be regarded as a fixed model without considering the sinkage and trim effects in the numerical simulation, the predicting results show the error was varied in an acceptable range. If the Froude Number is greater than 0.3, the difference between simulation and experimental results becomes wider, then the sinkage and trim effect acting on the resistance need to be considered in order to get more precise and satisfactory prediction results. So in this essay, a static-equilibrium based modification method is proposed to solve the problem of evaluating navigation gesture. By taking advantages of the ICEM CFD multi-block grids technology, the modification process can be executed without much extra work. The predicted value of sinkage and trim are basically accurate and acceptable. And the resistance results estimated based on this method agree pretty well with the experimental results.

\section{References}

1. A. T. P. Alho, "Validation of CFD Predictions of the Hull Resistance and the Wave System of a Catamaran." ASME 2012, International Conference on Ocean, Offshore and Arctic Engineering, 4 (2012):815-821.

2. O. Yaakob, Y. M. Ahmed, and M. R. Othman, "Use of CFD to Study the Resistance of Sprint Master Canoe." Applied Mechanics \& Materials, 695(2014):463-467.

3. H. C. Raven, A. V. D. Ploeg, and A. R. Starke. "Towards a CFD-Based Prediction of Ship Performance--Progress in Predicting Full-Scale Resistance and Scale Effects." International Journal of Maritime Engineering, 150(2008).

4. W.B. Yi, Y.S. Wang and Q.F. Yang, et al., "Numerical Methods for Predicting Ship Resistance and Flow Field." Harbin Gongcheng Daxue Xuebao/Journal of Harbin Engineering University, 35 .5(2014):532-536.

5. T. Tezdogan, A. Incecik, and O. Turan, "A numerical investigation of the squat and resistance of ships advancing through a canal using CFD." Journal of Marine Science \& Technology, 21.1(2016):81-101.

6. C.B. Ni et al., "A New Method to Predict Full Scale Ship Resistance Based on CFD Theories." The Twenty-second National Symposium on Hydrodynamics (2009).

7. C.Y. Guo, L.Z Wang, Q.X. Zhao. et al., " Approach of Ship Resistance Prediction with Consideration of Hull Gesture.” Ship Engineering, 35.01(2015):31-34. 
8. W.C Dong, C.B. Yao. "Study on resistance prediction method and resistance reduction mechanism of medium \& high speed Deep-Vee ship by stern flap." Journal of Harbin Engineering University, 32.07(2011).

9. R. Deng, et al., "Investigation on Some Factors Effecting Ship Resistance Calculation with CFD Code FLUENT.” Chuan Bo Li Xue/journal of Ship Mechanics, 17.06(2013):616-624.

10. H.W. Sun, W.J. Ma, and J.B. Zhu, "Research on Grid Factor in Numerical Calculation of Planing Craft Resistance.” Shipbuilding of China, 56.02(2015):170-178.

11. F.R. Menter, "Two-Equation Eddy-Viscosity Transport Turbulence Model for Engineering Applications.” Aiaa Journal, 32.8(1994): 1598-1605. 